\title{
Reactive Pericarditis post Meningococcal Vaccine: First Case Report in the Literature
}

\author{
Ebrahim Khalid Al-Ebrahim, ${ }^{(1)}$ Alaa Algazzar, ${ }^{(10}$ Mohammed Qutub ${ }^{(0)}$ \\ King Abdulaziz University, Jeddah - Saudi Arabia
}

\section{Introduction}

Autoimmune allergic reaction causing myopericarditis following vaccination is extremely rare and, in this regard, much attention has been paid to smallpox vaccine. ${ }^{1}$ Halsell et al., ${ }^{2}$ reported 18 cases of probable myopericarditis in 230,734 primary vaccines after smallpox vaccination (an incidence of 7.8 per 100,000 over 30 days). No cases of myopericarditis following smallpox vaccination were reported among 95,622 vaccines who were previously vaccinated. A causal relationship was supported by the close temporal clustering (7-19 days; mean, 10.5 days following vaccination) and wide temporal distribution, occurrence in only primary vaccinees, and lack of evidence for alternative etiologies associated with myopericarditis. ${ }^{2}$

The meningococci causing primary meningococcal pericarditis (PMP) are usually of serotype C (88\% of cases in one series) or, less commonly, serotypes B or W135. ${ }^{3}$ PMP is a purulent pericarditis caused by N. meningitidis, with no evidence of previous meningococcemia or meningitis, with development of cardiac tamponade in many cases. Evaluation of PMP versus viral pericarditis is important since the former usually requires pericardial drainage in addition to antibiotics.

\section{Case presentation}

\section{Chief complaints}

A 22-year-old Saudi male presented to the hospital emergency department with retrosternal chest pain,

\section{Keywords}

Meningococcal Vaccines/adverse effects; Pericarditis/ complications; Reaction. stiff neck, fatigue, and sudden fever. The patient had taken ibuprofen for symptomatic relief, and had no significant medical history.

\section{History of present illness}

The patient reported meningococcal vaccination (Meningococcal groups A, C, W-135 and Y conjugate vaccine) 5 days before admission.

\section{History of past illness}

No history of smoking, hypertension, diabetes, dyslipidemia or family history of premature coronary artery disease. Review of systems was unremarkable. The patient had no history of medication use or recreational drugs, or any medical preparations prior to admission. The patient was born in Saudi Arabia and his immunization status was up to date.

\section{Physical examination}

On physical examination, blood pressure was 130/70 $\mathrm{mm} \mathrm{Hg}$, heart rate was 95 beats/min, oral temperature $37.5{ }^{\circ} \mathrm{C}$ and the oxygen saturation $100 \%$ on room air. There was diffuse redness and tenderness over the vaccination site. Cardiac examination revealed normal jugular venous pressure (JVP), normal apical beat, normal S1 and S2 with no extra heart sounds, murmurs, or rubs.

\section{Laboratory tests}

Extensive evaluation of laboratory results found normal levels of lymphocytes, monocytes, and eosinophils. Other laboratory results showed elevated troponin, aspartate aminotransferase (AST), lactate dehydrogenase (LDH), brain natriuretic peptide (BNP), erythrocyte sedimentation rate (ESR) and C-reactive

Mailing Address: Ebrahim Khalid Al-Ebrahim

King Abdul Aziz University - Al Ehtifalat St. Postal Code: 21589, Jeddah - Saudi Arabia

E-mail: E.alebrahim97@gmail.com 
protein (CRP). Results of five consecutive days are described in Table 1.

The results of virus studies investigating other possible causes of myopericarditis were negative for HIV and hepatitis virus. Blood and urine cultures were negative Stool cultures were negative for enteroviruses. Serum electrolyte levels and chest radiography findings were normal.

\section{Imaging tests}

An initial electrocardiogram (ECG) showed diffuse concave upward ST-segment elevation on limb leads II, III, aVF, and chest leads V3, V4, V5, V6 (Figure 1), with PR segment elevation in lead aVR. An echocardiogram performed on admission revealed normal biventricular systolic function without regional wall motion abnormalities and trivial pericardial effusion and CT coronary angiography revealed normal coronaries.

\section{Final diagnosis}

The diagnosis of pericarditis was made based on the presence of fever, recent immunization and absence of risk factors for coronary artery disease with positive ECG changes and elevated cardiac markers.

\section{Treatment and hospital course}

The patient was admitted to the cardiac intensive care unit for observation and monitoring. A regimen of acetylsalicylic acid, $600 \mathrm{mg}$ orally every $6 \mathrm{~h}$, colchicine $0.5 \mathrm{mg}$ twice daily was started, and pantoprazole $40 \mathrm{mg}$ once daily was administered for gastric protection. The patient showed a dramatic clinical improvement after four days of therapy, with normalization of inflammatory and cardiac markers. The findings of an echocardiogram performed one week after discharge were normal.

\section{Outcome and follow-up}

After five days of treatment and at follow-up visits, for one month, the patient was asymptomatic, and has an uneventful clinical course.

\section{Discussion}

Perimyocarditis is an acute inflammation of the pericardium and the underlying myocardium resulting in myocellular damage. It can be considered an acute pericarditis with elevated cardiac biomarkers. Although cardiac biomarkers were elevated in our patient, the echocardiography showed normal myocardium and normal wall motion.

PMP is a rare form, usually presented as acute purulent pericarditis, that is complicated by cardiac tamponade and requires pericardial drainage. PMP is typically caused by Neisseria meningitidis of serotype C, or, less commonly, B or W135. In general, meningococcal myopericarditis is categorized into three etiologies;

Table 1 - Circulating biomarkers of the patient in five consecutive days

\begin{tabular}{|c|c|c|c|c|c|}
\hline Cardiac markers & $1^{\text {st }}$ day & $2^{\text {nd }}$ day & $3^{\text {rd }}$ day & $4^{\text {th }}$ day & $5^{\text {th }}$ day \\
\hline Troponin-I (ug/L) * & 16 & 9.90 & 6.46 & 4.16 & 0.02 \\
\hline Mass CK-MB (ug/L) & 30 & 25 & 16 & 2.5 & 1.3 \\
\hline NT-pro BNP (pig/ml) & - & 2107 & 1576 & 364 & 100 \\
\hline CRP (mg/L) & 90 & 135 & 103 & 55 & 13 \\
\hline $\operatorname{ESR}(\mathrm{mm} / \mathrm{h})$ & 34 & 74 & 80 & 44 & 7 \\
\hline AST (U/L) & 100 & - & 44 & 25 & - \\
\hline $\mathrm{LDH}(\mathrm{U} / \mathrm{L})$ & 387 & 414 & 356 & 324 & 200 \\
\hline \multicolumn{6}{|c|}{$\begin{array}{l}\text { - NT-pro BNP: N-terminal prohormone of brain natriuretic peptide. } \\
\text { - CRP: C reactive protein } \\
\text { - ESR: Erythrocyte sedimentation rate } \\
\text { - AST: Aspartate amino transferase. } \\
\text { - LDH: Lactate dehydrogenase. } \\
\text { - Normal Troponin level up to } 0.04 \mathrm{ug} / \mathrm{l}\end{array}$} \\
\hline
\end{tabular}




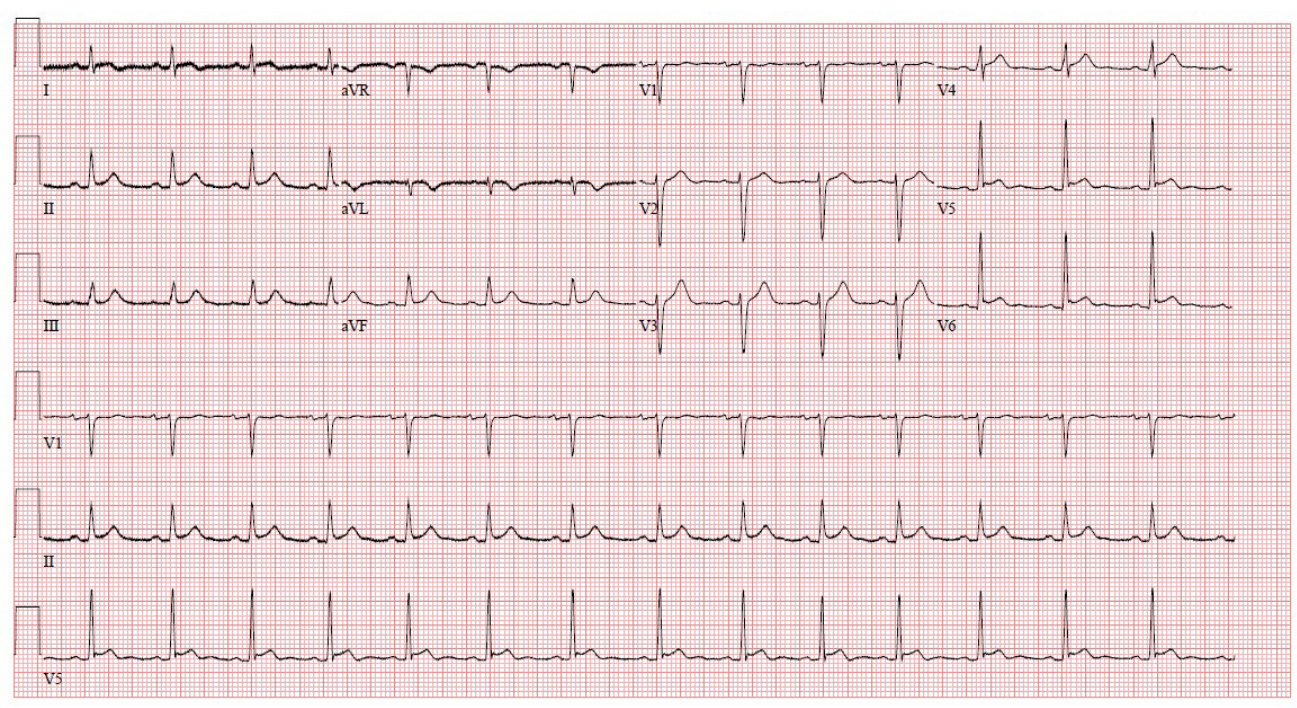

Figure 1 - Echocardiogram showing diffuse concave upward ST-segment elevation on limb leads II, III, aVF, and chest leads V3,V4, V5, V6 (Figure 1), with PR segment elevation in lead aVR

primary meningococcal disease, secondary disease due to disseminated meningococcemia, and reactive meningococcal pericarditis (RMP), an immunologic complication. ${ }^{4}$ Because of the negative history of infection, the exposure to meningococcal conjugate vaccine (groups $\mathrm{A}, \mathrm{C}, \mathrm{W}-135$ and $\mathrm{Y}$ ) five days prior to the onset of symptoms, and negative serology for infectious and autoimmune diseases, we concluded that pericarditis was reactive rather than a result of direct infection of the myocardium, and a combination of acetylsalicylic acid and colchicine was initiated.

Many case reports of RMP following meningococcal infection have been reported, but no case report of RMP following meningococcal vaccination has been published. RMP is a rare condition and may be more severe than purulent pericarditis; cardiac tamponade is relatively common, requiring high doses of steroids and/or pericardiocentesis. RMP is usually seen 6-15 days after the onset of illness and is characterized by a type III hypersensitivity reaction, either against the specific serotype of the N. meningitidis or against a newly antigenic, damaged pericardial tissue because of molecular mimicry with microbial antigens. Contrary to purulent pericarditis, RMP represents a late complication and there have been many reported cases of RMP post-meningococcal infection in global literature since 1969 (Table 2). ${ }^{5-10}$ Severe disease, age (adults and young teenagers), and serogroup C seem to predispose to post-infectious, immune-related complications including arthritis, vasculitis, pleuritis, and pericarditis. ${ }^{7-9}$ Recently, much attention has been given to smallpox vaccine due to its association with myocarditis. ${ }^{1}$ There is one reported case of myocarditis that developed hours after diphtheria, tetanus, and acellular pertussis $(\mathrm{DTaP})$ vaccination in a 3-monthold infant, ${ }^{11}$ and another case of myocarditis after tetanus vaccination in a 14-year-old child. ${ }^{12}$ In line with these observations, our patient was a young adult, presenting in poor clinical condition, elevated inflammatory markers suggestive of severe disease, and responsive to anti-inflammatory drugs without antibiotics. We considered the diagnosis of reactive pericarditis rather than myopericarditis due to normal echocardiography and a conservative course of management. The clinical data presented in the case report support the etiologic relationship between meningococcal vaccination and pericarditis. It may be an association, or even a causative relationship. Taking the risk of myocardial biopsy in consideration, histopathological diagnosis of the myocardial biopsy will show diffuse inflammation and will not specify the exact meningococcal etiology.

\section{Conclusion}

Further observations and studies are needed to support this case report to prove a causative role of meningococcal vaccine in pericarditis. 
Table 2 - Previous case reports of reactive meningococcal pericarditis in the literature

\begin{tabular}{|c|c|c|c|c|c|}
\hline Reference & $\begin{array}{c}\text { Patient } \\
\text { age/y }\end{array}$ & $\begin{array}{c}\text { Time of } \\
\text { pericarditis } \\
\text { diagnosis }\end{array}$ & $\begin{array}{c}\text { Neisseria } \\
\text { meningitidis } \\
\text { serogroup }\end{array}$ & Clinical presentation & Therapy \\
\hline Chiappini ${ }^{5}$ & 10 & $7 \mathrm{~d}$ & C & Meningitis & Prednisone + Aspirin \\
\hline El Bashir ${ }^{6}$ & 13 & $7 \mathrm{~d}$ & C & Meningitis & $\begin{array}{c}\text { Dexamethasone + Ibuprofen, later } \\
\text { diclofenac sodium }\end{array}$ \\
\hline Dupont $^{7}$ & 14 & $3 \mathrm{~d}$ & C & Meningitis & ASA \\
\hline Stephani ${ }^{8}$ & 14 & $9 \mathrm{~d}$ & C & $\begin{array}{c}\text { Meningitis, } \\
\text { endophthalmitis }\end{array}$ & Prednisone + Antibiotics \\
\hline $\begin{array}{l}\text { Fuglsang } \\
\text { Hansen }{ }^{9}\end{array}$ & N/A & $11 \mathrm{~d}$ & N/A & Meningitis & Steroid + Pericardiocentesis \\
\hline Akinosoglou $\mathrm{K}^{10}$ & 28 & N/A & B & $\begin{array}{l}\text { Meningococcemia and } \\
\text { acute abdominal pain }\end{array}$ & Steroid + Antibiotics \\
\hline
\end{tabular}

Patients who have chest complaints after vaccination should be evaluated for post-vaccine pericarditis and myocarditis. High clinical suspicion, careful examination and close observation may be required so that atypical presentation and manifestations are not overlooked. Endomyocardial biopsy specimens might help to determine the relative causative role of vaccine in the development of myocarditis. In our case, the mild illness, uncomplicated course, and good response to treatment deferred the need for biopsy. To our knowledge, there is no case report of reactive pericarditis following meningococcal vaccination.

\section{Author contributions}

Conception and design of the research: Al-Ebrahim EK, Qutub M. Analysis and interpretation of the data: Algazzar A. Writing of the manuscript: Al-Ebrahim EK. Critical revision of the manuscript for intellectual content: Algazzar A, Qutub M.

\section{Limitation of the report}

In these cases, in order to find the cause/effect relationship (or the association), large epidemiological studies are needed.

\section{Potential Conflict of Interest}

No potential conflict of interest relevant to this article was reported.

\section{Sources of Funding}

There were no external funding sources for this study.

\section{Study Association}

This study is not associated with any thesis or dissertation work.

\section{Ethics approval and consent to participate}

This article does not contain any studies with human participants or animals performed by any of the authors. 


\section{References}

1. Arness MK, Eckart RE, Love SS, Atwood JE, Wells TS, Engler RJ, Collins LC, Ludwig SL, Riddle JR, Grabenstein JD, Tornberg DN. Myopericarditis following smallpox vaccination. Am J Epidemiol 2004;160(7):642-51.

2. Halsell JS, Riddle JR, Atwood JE, Gardner P, Shope R, Poland GA, Gray GC, Ostroff S, Eckart RE, Hospenthal DR, Gibson RL, Grabenstein JD, Arness MK, Tornberg DN; Department of Defense Smallpox Vaccination Clinical Evaluation Team. Myopericarditis following smallpox vaccination among vacciniaive US military personnel. JAMA 2003;289(24):3283-9.

3. Hardy DJ, Bartholomew WR, Amsterdam D. Pathophysiology of primary meningococcal pericarditis associated with neisseria meningitidis group C. A case report and review of the literature. Diagn Microbiol Infect Dis. 1986;4(3):259-65.

4. Finkelstein $Y Y$, Adler $Y$, Nussinovitch M. A new classification for pericarditis associated with meningococcal infection. Eur J Pediatr. 1997;156:585-8.

5. Chiappini E, Galli L, de Martino M, De Simone L. Recurrent pericarditis after meningococcal infection. Pediatr Infect Dis J 2004;23(7):692-3.
6. El Bashir H, Klaber R, Mukasa T, Booy R. Pericarditis after meningococcal infection: case report of a child with two distinct episodes. Pediatr Infect Dis J. 2004; 23(3)23: 279-81.

7. Dupont M, du Haut Cilly FB, Arvieux C, Tattevin P, Almange C, Michelet C. Recurrent pericarditis during meningococcal meningitis. 2 case reports. Presse Med 2004;33(8):533-4

8. Stephani U., and Bleckmann H.: Rare complications in a case of generalized meningococcal disease: immunologic reaction versus bacterial metastasis. Infection 1982;10(1):23-7.

9. Fuglsang Hansen J., Johansen I.S.: Immune-mediated pericarditis in a patient with meningococcal meningitis. Ugeskr Laeger. 2013;175(14):967-8.

10. Akinosoglou K, Alexopoulos A, Koutsogiannis N, Gogos C, Lekkou A.Neisseria meningitidis presenting as acute abdomen and recurrent reactive pericarditis. Braz J Infect Dis 2016;20(6):641-4.

11. Cassimatis DC, Atwood JE, Engler RM, Linz PE, Grabenstein JD, Vernalis MN. Smallpox vaccination and myopericarditis: a clinical review. J Am Coll Cardiol. 2004;43(9):1503-10.

12. Dilber E, Karagoz T, Aytemir K,Ozer S, Alehan D, Oto A. Acute myocarditis associated with tetanus vaccination. Mayo Clin Proc. 2003;78(11):1431-3. 